\title{
A study of bonding to moist enamel using composite resins
}

\author{
The bonding of composite resin to moist enamel by A. W. G. Walls, J. Lee and J. F. McCabe \\ Br Dent J 2001; 191: 148-150
}

\author{
Objective \\ To determine the effect on the bond strength of modern dentine \\ bonding agents to etched enamel of surface contamination with \\ water.
}

\section{Design}

Fifteen bond strength measurements were made for bonds prepared to both moist and dry etched enamel for each of three test and one control materials.

\section{Results}

For two materials (Scotchbond 1 and Prime and Bond 2.1) the bond strength was not affected by the presence of water on the etched enamel surface. A mean bond strength in excess of $25 \mathrm{MPa}$ was achieved for both materials under all conditions. One material (Optibond Solo) showed a 30\% increase in bond strength when bonds were formed under wet condition $(21.10$ $\mathrm{MPa}$ compared with $15.35 \mathrm{MPa}$ ). The bond strength of the control material, a conventional unfilled bonding resin, decreased markedly with aqueous contamination $(9.14 \mathrm{MPa}$ compared with $26.75 \mathrm{MPa}$ ).

\section{Conclusion}

Etched enamel should be rehydrated routinely prior to bonding composite resin to its surface using a water displacing dentine bonding system.
In Brief
- Bonding resins to damp dentine necessitates the bonding also to damp enamel. This paper explores the ability of dentine bonding systems to bond to damp etched enamel.
- All of the 'water chasing' dentine bonding systems used in this study were capable of bonding to damp etched enamel, at least as effectively as to dry etched enamel.
- The classical unfilled resin system used as a control suffered a $66 \%$ fall in bond strength when the etched enamel surface was contaminated with water.

\section{Comment}

Cor many years dentists and dental mateFrial scientists have been concerned with the problems of bonding to dentine and how to achieve adhesion to a wet substrate. Adhesion of materials to enamel is taken almost for granted and has allowed the development of many forms of resinretained restorations.

In the past, adhesion to dentine was poor because the bonding materials did not wet the solid surfaces. This poor surface wettability also meant that voids formed and thus further reduced adhesion. It is therefore of advantage to have adhesives that either displace water or react with or absorb that water.

The introduction of such adhesives means that reliable adhesion to dentine can be obtained but only if the dentine is moist. This poses a dilemma to most dentists as we have been brought up to believe that enam- el should be dry (if not desiccated) to get a good bond. We all like to see the frosty appearance of etched enamel as this confirms that we have etched the tooth.

But how are we to get reliable bonding to both dentine and enamel if one has to be wet and the other dry? Its just not possible to do clinically. Fortunately these authors have answered the question. The enamel can be moist like the dentine and the bond will still be adequate. This study looked at three current dentine bonding systems and in all cases the bond was at least as strong on moist enamel as on dry. Moist enamel was achieved by wetting the enamel with water after etching, then blotting with a cotton wool pledget to leave a damp surface.

Was the bond strong enough? It is generally believed that bonds should be $17-20$ MPa to resist polymerisation shrinkage of the restorative material and to retain the restoration in the cavity. Such bond strengths were achieved in the laboratory tests in this study. The ideal test for any material or restorative system would be a clinical trial over a number of years, however this is rarely possible in the current dental materials market. Laboratory tests can give an indication of the potential performance of new products and allow comparison of a number of products.

This neat study has answered a question that has confused many dentists since the concept of wet bonding was introduced. Now we know that both enamel and dentine may be 'wet' and adequate bond strengths will be achieved with current bonding systems.

\section{Dr Ann C. Shearer}

Lecturer in Primary Dental Care, University

Dental Hospital of Manchester 